\title{
Políticas de igualdad de género en los Ayuntamientos de Barcelona: origen, características y retos para el futuro*
}

\section{Dra Emanuela Lombardo**}

\section{Introducción}

El tema de este ensayo son las políticas de género locales, con particular referencia a los Ayuntamientos de la provincia de Barcelona. El análisis se basa tanto en la literatura relativa al tema tratado como en un trabajo empírico mediante entrevistas telefónicas a algunos Ayuntamientos de la provincia de Barcelona. La extensión de competencias de las administraciones municipales en asuntos de igualdad entre mujeres y hombres se realiza en el marco de la Ley 7/1985, de 2 de abril, Reguladora de las Bases del Régimen Local. Esta extensión es parte de un fenómeno más general de progresivo impulso del ámbito local, conocido como el 'nuevo localismo', que se caracteriza como respuesta de las comunidades locales a los desafíos que genera la creciente globalización económica, política y cultural. Asimismo, el concepto de 'subsidiariedad' recogido en el Tratado de Maastricht de 1993 ha conferido mayor legitimidad al poder de las administraciones municipales, según el principio de que las decisiones las deben tomar las instituciones más cercanas a los ciudadanos.

El ámbito de las políticas de género se ha beneficiado de la ampliación de competencias municipales, y a partir de los años noventa se ha desarrollado progresivamente en muchos Ayuntamientos de las provincias españolas. Este proceso ha favo- recido un aumento del interés académico hacia el nivel local de las políticas de género, que apenas se había estudiado anteriormente en España. Este ensayo se propone precisamente ampliar el conocimiento de las políticas de género locales a través del análisis de distintos aspectos de las mismas: las razones de su origen y desarrollo, las características de los organismos locales de igualdad y de las medidas políticas que se llevan a cabo, la actividad de los centros de atención a mujeres, la aplicación del mainstreaming de género o transversalidad por parte de las administraciones locales ${ }^{1}$.

En la primera sección se explora el origen, tanto histórico como político, de las medidas públicas para favorecer la igualdad de género y se exponen las valoraciones principales del feminismo institucional que nos ofrece la literatura sobre género. La segunda parte analiza el desarrollo de las políticas de igualdad en España, tanto en el ámbito central como en el autonómico, con referencia a los planes como instrumentos privilegiados de acción para la igualdad en el territorio español. El marco teórico y legal de las políticas de género locales es el objeto de la tercera sección, mientras que la cuarta parte se centra en un estudio del caso de los Ayuntamientos de la provincia de Barcelona, realizado mediante entrevistas telefónicas semi-estructuradas a las responsables de nueve concejalías de la mujer. La quinta sección se ocupa específicamente de los centros de atención directa a mujeres, analizando sus características y sus límites. La aplicación de la transversalidad en los Ayuntamientos de Barcelona es el obje- 
to de la sexta sección, en la que se mencionan los obstáculos existentes a la coordinación interdepartamental y se sugieren algunas recetas que los mismos municipios han adoptado para implementar la transversalidad.

Las conclusiones indican algunos de los retos para el futuro de las políticas de género locales. Uno de estos retos es articular actuaciones globales dirigidas a prevenir las discriminaciones más que a curarlas y a promover la autonomía de la mujer en lugar de la dependencia, con el fin de que las políticas puedan superar un enfoque asistencialista hacia las mujeres. Otro reto fundamental consiste en un empleo eficaz de la red de relaciones, debido a que numerosas innovaciones de las perspectivas de género y de las estrategias de acción de los organismos locales provienen de los contactos entre los distintos niveles y foros gubernamentales y no gubernamentales. Parte del mismo desafío es la coordinación horizontal del trabajo, para la cual hay que superar los obstáculos puestos tanto por las delimitaciones institucionales como por la resistencia humana al cambio de las pautas de trabajo y de relación. Un último objetivo hacia el cual deberían trabajar las políticas de género locales es dar más espacio a la participación de las mujeres.

\section{Origen de las políticas de igualdad}

Las 'políticas de igualdad' se pueden definir como el conjunto de las decisiones, objetivos y medidas adoptadas por las instituciones públicas en relación al fomento de la igualdad entre mujeres y hombres y a la mejora de la situación socio-económica, política y cultural de la mujer. El punto de partida de estas políticas públicas de igualdad se tiene que buscar en las luchas de los movimientos de mujeres de la denominada 'segunda ola' del feminismo, que ocurre en las décadas de los sesenta y setenta. En cualquier discurso sobre derechos de la mujer es preciso tener presente el compromiso tomado por estos colectivos de mujeres y la presión que éstos ejercieron en momentos fundamentales de elaboración de medidas normativas y políticas.

Uno de los resultados de los movimientos feministas contemporáneos ha sido el establecimiento de un cierto consenso político sobre la legitimidad de las demandas de las mujeres. Se empieza a reconocer que los poderes públicos deben actuar para atenuar las desigualdades de género y la discriminación de la mujer. Esto causa que un cierto número de países empiecen a incluir en sus políticas públicas medidas para combatir la discriminación sexual y promover la igualdad entre mujeres y hombres. La mayoría de los países europeos introdujeron sus primeras medidas legales de igualdad y no discriminación en sus respectivas Constituciones después de la segunda guerra mundial. A partir de los años setenta se crearon en los Estados occidentales organismos de igualdad, es decir, instituciones cuya función es impulsar políticas para fomentar la igualdad entre mujeres y hombres. Esto es lo que se conoce como 'feminismo de Estado' (STETSON y MAZUR, 1995) o feminismo institucional' (LovendusKI, 1997). En otras opiniones (Threlfall, 1998; Gelb, 1989), es mejor hablar de 'feminismo al gobierno' por dos razones. La primera es que las políticas de igualdad están especialmente sujetas a los cambios de partido en el gobierno, nacional o local, que puede abolirlas, mantenerlas o modificarlas. La segunda razón es que el contenido feminista de las políticas de igualdad es muy dependiente del éxito de la relación entre activistas feministas y partido/s en el poder, debido al papel de los partidos de controlar el acceso a los recursos de los diferentes niveles de administración pública.

De la forma en la que se quiera denominar, esta respuesta de los poderes públicos a las demandas de igualdad y no discriminación de los movimientos de mujeres ha recibido dos valoraciones distintas por parte de las activistas y de las académicas, una menos y otra más favorable a la creación de políticas públicas de igualdad (VALIENTE, 1996). El feminismo más radical considera la institucionalización como una imposición 'desde arriba' que limita el potencial crítico del movimiento (HERNES, 1990: 23). Critica también la efectividad de las medidas de igualdad que se pueden conseguir a través de las políticas institucionales, debido a la escasez de los recursos de poder económico y político de los organismos de igualdad, que sólo pueden intentar convencer a los otros poderes públicos para que promuevan políticas de igualdad (VALIENTE, 1998/1999).

A pesar de estos límites, muchos estudios, entre los cuales cabe destacar el primer estudio comparativo internacional sobre feminismo oficial en 14 Estados (Alemania, Australia, Canadá, Dinamarca, España, EEUU, Francia, Irlanda, Italia, Noruega, Países Bajos, Polonia, Reino Unido y Suecia), valoran los organismos de igualdad por su capacidad de impulsar leyes y políticas para el avance de las mujeres y aplicar los objetivos del movimiento feminista con medidas políticas concretas (STETSON y MAZUR, 1995). El estudio comparativo subraya dos elementos clave para aumentar la efectividad de las instituciones de igualdad: una opinión pública que apoye las intervenciones estatales en materia de igualdad (con aportaciones de la sociedad civil a la elaboración de las políticas de género) y unos movimientos feministas fuertes que colaboren con los organismos de igualdad (VAliente, 1998/1999). 


\section{Políticas de igualdad en España: el nivel central y autonómico}

En España, el paso hacia el establecimiento de 'políticas de igualdad' fue dado con la creación del Instituto de la Mujer (IM) en 1983 (Ley 16/1983), debido a presiones ejercidas tanto por la influencia internacional de NNUU, la Comunidad Europea y el resto de los Estados miembros, como del movimiento feminista dentro y fuera de España, y sobre todo, por las presiones de las activistas socialistas dentro del neo-electo PSOE en 1982 (Threlfall, 1994; Instituto de la Mujer, 1994; VALIENTE, 1996; GiL RuIz, 1996).

A partir de entonces, se empiezan a elaborar medidas para conseguir una igualdad no solamente formal sino también sustancial, a través del instrumento de los planes. En 1987 se aprueba el I Plan de Igualdad de Oportunidades de las Mujeres (19881990), que se inspira en el modelo de los dos primeros Programas de Acción Comunitaria (1982-1985 y 1986-1990), y continúa con el II Plan (1993-1995), que se inserta en el marco del Tercer Programa de Acción Comunitaria (1991-1995) y el III Plan (1997-2000), que se inserta en el Cuarto Programa Marco Comunitario (1996-2000) y el IV Plan (2003-2006), que se inspira en el Quinto Programa Marco Comunitario (2001-2005).

El IM, que actualmente es un organismo autónomo dentro del Ministerio de Trabajo y Asuntos Sociales, no tiene capacidad para implementar directamente políticas de igualdad, sino que debe persuadir a los diferentes Ministerios y departamentos para que estos incluyan políticas de igualdad en sus agendas y las lleven a cabo. El IM nacional tiene competencia y presupuesto para desempeñar esencialmente tres tareas:

investigación sobre la situación de las mujeres en España;

información para las mujeres sobre sus derechos;

canalización de denuncias formuladas por mujeres a las instituciones competentes.

La caracterización del IM como impulsor de políticas de igualdad más que como ejecutor directo, se indica como razón que está detrás de la adopción por parte del Instituto de los planes de igualdad como instrumento principal para desarrollar políticas de igualdad (BUSTELO, 2002). Es decir, como los organismos no tienen ni recursos ni poder para ejecutar medidas, recurren a los planes, que a su vez, dependen de las capacidades persuasivas de las femócratas.

\section{El instrumento fundamental: los planes de igualdad}

Los planes de igualdad se definen como 'un conjunto de objetivos y medidas tomadas y aprobadas por un gobierno y que deben llevar a cabo los diferentes departamentos gubernamentales, en un período concreto de tiempo (de dos a cinco años).' (Bustelo, 2002: 52). Teniendo en cuenta los escasos recursos que tienen los organismos de igualdad, los planes aumentan las posibilidades de introducir horizontalmente la perspectiva de género en todas las áreas gubernamentales, debido a que requieren actuaciones conjuntas por parte de los distintos departamentos y sectores gubernamentales.

BusTelo destaca otras ventajas de los planes, entre las cuales se encuentran el hecho de que promueven tanto la conciencia de los poderes públicos sobre igualdad de género como su compromiso a través de la aprobación del plan en Consejo de Ministros o de Gobierno; la posibilidad de tratar el problema de la igualdad desde una perspectiva global e integral en lugar de con actuaciones sectoriales; la coordinación interdepartamental; el establecimiento de objetivos estratégicos para cuya realización hay que prever recursos y compromisos; el seguimiento y la evaluación de los planes, que permite la mejora de los mismos; la participación de asociaciones de mujeres en la fase precedente a la elaboración del plan; el apoyo recibido por parte de Naciones Unidas (Plataforma de Pekín) y Unión Europea (Cuarto Programa de Acción); y la posibilidad de representar un modelo para las políticas de igualdad de género locales (BusTelo, 2002).

Estas razones pueden explicar que los planes hayan tenido tanto éxito como instrumentos de actuación del feminismo institucional en España. Sin embargo, las expertas señalan también el riesgo de que los planes terminen teniendo un valor simbólico siempre más avanzado, pero un grado de ejecución y un impacto mínimos (Sensat y Varella, 1998; Bustelo, 2002).

\section{El marco autonómico}

La gestión de las políticas de igualdad en España se realiza de forma 'semi-descentralizada', a través de los organismos de igualdad que actúan en los tres niveles de la Administración estatal, es decir el central, el autonómico y el local (SENSAT y VarelLa, 1998: 357). El proceso de creación de las comunidades autónomas ha implicado, entre otras, la descentralización de las políticas de igualdad ${ }^{2}$. Por lo tanto, en cada CA se han instituido organismos parecidos al IM central con el fin de promover medidas de igualdad y no discriminación por parte de las instituciones autonómicas, y se han desarrollado planes de igualdad según el modelo central (AstelarRA, 1996). A partir del 1988, los organismos de igualdad se difunden en todas las CCAA (con la excepción de Ceuta y Melilla), y los planes se empiezan a lanzar poco después de las creaciones de los institutos. Se habla de un 
'efecto cascada' de los organismos de igualdad y del instrumento de los planes, debido a la difusión relativamente rápida de las medidas ${ }^{3}$.

Sin embargo, a pesar de la uniformidad en la creación de organismos específicos de igualdad de género en el nivel autonómico y en el uso de Planes como instrumentos estratégicos para actuar, existe una variedad considerable en cuanto al marco institucional de los distintos organismos. Como analiza Bustelo, la diferencia de tipo de organismo (autónomo u otro) y la posición del organismo de igualdad en el organigrama del respectivo gobierno (a qué departamento gubernamental pertenece) son factores determinantes, tanto para el grado de autonomía del instituto como para promover medidas transversalmente en todas las áreas de gobierno en lugar de limitar el impulso a las áreas específicas (familia, bienestar social, empleo, etc.) donde está adscrito (Bustelo, 1999; 2002).

El Institut Catalá de la Dona, por ejemplo, que se crea con la Ley 11/1989, de 10 de julio, tiene dos ventajas, junto a otros pocos organismos autonómicos: en primer lugar, es un organismo autónomo y, en segundo lugar, depende del departamento de Presidencia. Las ventajas de ser un organismo autónomo consisten en lo siguiente: es creado por ley y no por decretos, como la mayoría de los organismos; tiene su propio presupuesto; tiene personalidad jurídica propia y una mayor autonomía administrativa; tienen un Consejo de Administración en el que pueden ser representadas otras fuerzas sociales. La dependencia de la Presidencia es un factor positivo en cuanto a la capacidad del instituto de actuar transversalmente para convencer a los departamentos a poner en practica las medidas de los planes de igualdad e introducir la perspectiva de género de forma más eficaz en todas las áreas (BUSTELO, 2002).

\section{El mainstreaming de género en los niveles central y autonómico}

La estrategia política más reciente en materia de género es conocida como mainstreaming, que en castellano se ha traducido con el termino 'transversalidad' ${ }^{4}$. Éste se refiere a la introducción por parte de los gobiernos y otros actores institucionales de una perspectiva de género en todas las políticas y los programas, con la intención no sólo de analizar sus efectos sobre las mujeres y los hombres antes de tomar decisiones, sino también de implementar, evaluar y revisar políticas teniendo en cuenta la dimensión de género. El mainstreaming fue adoptado oficialmente en el ámbito de las políticas de género por primera vez en ocasión de la Conferencia mundial de las Naciones Unidas sobre mujeres en Pekín y, a partir de esta fecha, se introdujo en los nuevos artículos 2 y 3 del Tratado de Amsterdam de la Unión Europea y se ha reflejado en el Cuarto y Quinto Programa de Acción para
Igualdad de Oportunidades entre hombres y mujeres de la Comisión Europea (1996-2000 y 2001-2005).

En las políticas de igualdad de género en el ámbito central, la transversalidad se ha pretendido realizar a través de unos mecanismos de coordinación horizontal con los diversos departamentos ministeriales en las áreas que, según los planes, implicaban a las mujeres. Esta coordinación interministerial se ha realizado mediante convenios bilaterales y multilaterales y acuerdos de colaboración. Sin embargo, en la práctica, la coordinación horizontal se ha traducido en encargar a una persona de coordinar y seguir las actuaciones correspondientes de cada departamento o crear comisiones de trabajo en las que el mismo Instituto de la Mujer se encargaba de coordinar y seguir las actuaciones en el caso que varios Ministerios estuviesen involucrados (SENSAT y Varella, 1998). En la opinión de Sensat y Varella (1998), esta cooperación interministerial no resulta eficaz para aplicar la transversalidad de la perspectiva de género porque depende de la voluntad política de ministros y ministras. A pesar de que aún faltan estudios generales y profundizados para comprobar el grado de aplicación del mainstreaming de género en España, creo que difícilmente la realidad española se distingue de la situación actual común a muchos países europeos en los que la puesta en práctica de la transversalidad choca con dificultades que dependen tanto de las características jerárquicas de la estructura institucional como de elementos patriarcales propios de los mismos contextos políticos que deberían promoverla.

\section{Políticas de igualdad en el nivel local: marco teórico, origen y características}

Si los niveles nacional y autonómico tienen el papel de legislar e implementar medidas de igualdad, teniendo en cuenta las líneas estratégicas establecidas por las Naciones Unidas y la Unión Europea, es el ámbito municipal el que tiene un contacto más directo con la población y que puede detectar sus necesidades y prestar los servicios adecuados.

\section{El marco teórico}

A pesar de la importancia del papel municipal en este asunto, la bibliografía sobre políticas de igualdad es más extensa en lo que concierne los niveles central y autonómico, y carece mucho en lo que se refiere al ámbito local. Esta carencia depen- 
de de la escasa atención que han recibido hasta los años noventa las políticas públicas locales, debido a los fenómenos de 'nacionalización' (los gobiernos municipales no son políticamente relevantes porque dependen de instancias supra-municipales para establecer prioridades y líneas de actuación) y 'gerencialismo' (los gobiernos locales no son relevantes porque concentran todos sus esfuerzos en tareas ejecutivas). Ahora que la nacionalización empieza a ser superada por el fenómeno del 'nuevo localismo', es decir, por el creciente impulso al ámbito local que caracteriza esta época de globalización y debilidad de la soberanía de los Estados nacionales, y el 'gerencialismo' empieza a ser sustituido por una mayor politización y capacidad estratégica de los gobiernos locales, la investigación está empezando a dedicar una mayor atención a las políticas públicas locales (Equipo de Análisis Política, 2000).

Por lo que concierne las políticas de igualdad, entre otros, existe un estudio de la Federación Española de Municipios y Provincias realizado por SAMPEDRO que analiza las actuaciones de las Administraciones locales en el campo de la igualdad de género a través de los resultados de una encuesta cuantitativa dirigida a la totalidad de los municipios mayores de 20.000 habitantes, las Diputaciones provinciales, las CCAA, y una muestra de municipios de 5000 a 20000 habitantes (SAMPEDRO, 1992). A éste hay que añadir la contribución de Celia VALIENTE que consiste en un análisis de las políticas de igualdad de género en los Ayuntamientos de la Comunidad Autónoma de Madrid que utiliza una metodología más bien cualitativa con entrevistas en profundidad a personalidades de nueve municipios seleccionados en función del partido en el gobierno y de su tamaño (VALIENTE, 1998/1999).

\section{El marco legal}

El marco legal principal de las políticas de género locales está representado por la Ley 7/1985, de 2 de abril, Reguladora de las Bases del Régimen Local, en la cual se establece que tanto los municipios como las diputaciones pueden realizar actividades complementarias con otras Administraciones públicas, en particular en lo que concierne a la promoción de la mujer. La Ley Reguladora del Régimen Local, además, sanciona la competencia municipal en materia de servicios sociales y la obligación de su prestación en municipios mayores de 20.000 habitantes. La idea detrás de esta ley es que la posición de cercanía de la Administración local a la ciudadanía hace que este nivel sea el más adecuado para tomar medidas de carácter social, debido al mejor conocimiento de la realidad local. Apoyándose tanto en la Ley del Régimen Local como en los artículos de la Constitución española que establecen la igualdad jurídica (art. 14) y la obligación de los poderes públicos de promover la igualdad efectiva entre las personas (art. 9.2), las Administraciones locales han empezado a desarrollar políticas de igualdad de género para favorecer la promoción de la mujer.

\section{Los factores explicativos del origen y de la evolución de las concejalías de la mujer}

En los municipios se nota la tendencia hacia programas estables de atención a la mujer, creación de estructuras técnicas especificas, y de órganos políticos como por ejemplo las concejalías de la mujer, que son las responsables institucionales del plan o programa municipal de igualdad (SAMPEDRO, 1992). La existencia de concejalías en lugar de áreas de la mujer es una ventaja para la realización de políticas de igualdad debido a que la concejala, al ser miembro del equipo de gobierno, puede defender allí en primera persona las medidas que se tienen que tomar en el área de igualdad (VALIENTE, 1998/1999).

Entre los factores que se consideran más relevantes a la hora de explicar la creación de organismos de igualdad en las corporaciones locales se suelen incluir, en orden decreciente de importancia:

1) el tamaño del municipio (los núcleos de población más grandes de 15.000 habitantes suelen tener concejalía de la mujer, también debido a que la Ley Reguladora del Régimen Local, al sancionar la competencia municipal en materia de servicios sociales, prescribe la obligación de su prestación en municipios mayores de 20.000 habitantes);

2) el partido en el gobierno (el color político del partido ha sido importante en algún caso, pero no en todos, con un porcentaje mayor de concejalías en los municipios gobernados por partidos de izquierda);

3) el movimiento de mujeres (de impacto mínimo en la creación de concejalías de la mujer, o porqué no existía, o bien porque no tenía interés en el desarrollo del feminismo institucional) (SAMPEDRO, 1992; VAlieNTE, 1998/1999).

El presupuesto y la plantilla de la concejalía normalmente aumentan según el tamaño del municipio. Como en todos los organismos de igualdad del mundo, el presupuesto de las concejalías es insuficiente para llevar a cabo las medidas necesarias.

La plantilla de estos centros varía mucho. En ciertos casos hay una concejala que puede contar con un personal de apoyo, mientras que en otros casos la concejala es la única persona que se ocupa de políticas de igualdad, y que además es responsable de otras áreas como por ejemplo cultura, sanidad, y/o servicios sociales (SAmpedro, 1992; VAliENTE, 1998/1999).

Hay que destacar también la difusión de consejos de la mujer, es decir, órganos de participación de colectivos y asociaciones de mujeres que tienen una función consultiva para la Administración local y hacen un seguimiento de las actuaciones 
locales en materia de igualdad. Estos consejos dan más legitimidad a los organismos de igualdad, ya que permiten recoger las demandas de colectivos femeninos locales e incluirlas en el proceso de toma de decisiones.

\section{Las nuevas estrategias municipales en materia de igualdad}

Según SAMPEDRo (1992), se nota un cambio de enfoque en las líneas de actuación de las Administraciones Públicas en relación a los servicios sociales, que desde las practicas de asistencialismo y de atención individual se van orientando hacia un 'universalismo comunitario' que pretende fomentar la calidad de vida de la comunidad a través de medidas de acción preventivas y globales (Equipo de Análisis Política, 2000).

Las políticas de igualdad de género locales parecen seguir el mismo camino, ya que paralelamente a las intervenciones más personalizadas a mujeres se comienzan a desarrollar medidas estratégicas de carácter integral y preventivo, que requieren una mayor coordinación entre las distintas áreas municipales (Bienestar Social, Educación, Cultura, Salud, Empleo, Urbanismo y Vivienda, etc.). Esta exigencia está planteada por las mismas profesionales de servicios sociales, quienes insisten en el hecho de que la discriminación de la mujer no se agota en los problemas de marginación grave o situaciones limite. El programa de la mujer debe necesariamente contemplar diferentes niveles de intervención, distinguiendo distintos colectivos de mujeres según el tipo de atención que se les debe prestar, incluyendo el nivel de atención más amplio o global, como victimas de una situación de discriminación histórica que se refleja en todos los ámbitos de la vida cotidiana. La necesidad de una actuación de tipo integral, que transciende en muchas ocasiones las competencias y la capacidad operativa real del sistema de Servicios Sociales, es la consecuencia lógica de esta constatación.' (SAMPEDRO, 1992: 30)

\section{Los planes de igualdad municipales}

El resultado de este cambio de enfoque hacia una actuación más integral y preventiva ha sido la elaboración de planes o programas de igualdad municipales ${ }^{5}$ parecidos a los que se desarrollan en los niveles Europeo, nacional, y autonómico. Además, los planes se consideran actualmente como el instrumento más eficaz para poner en práctica la transversalidad, debido a dos factores principales:

1) el Plan, en la mayoría de los casos, tiene que aprobarlo el pleno municipal en su conjunto, y esto conlleva una asunción de responsabilidad política por parte del gobierno local hacia el problema de la desigualdad de género que es indispensable para respaldar la labor del departamento de la mujer;
2) las medidas de fomento de la igualdad de género articuladas en el plan tienen que ponerlas en marcha distintas áreas y departamentos municipales (y no solamente la concejalía de la mujer), promoviendo así la realización de una política intersectorial que evite solapamientos y acciones descoordinadas.

Estas características del plan facilitan en principio la adopción de la perspectiva de género horizontalmente en todas las áreas políticas, que es típica de la transversalidad. Sin embargo, en la práctica, las dificultades de coordinación interdepartamental y los obstáculos culturales e institucionales han favorecido la realización de políticas de género por parte de las concejalías de la mujer, pero no por parte de otras concejalías (VALIENTE, 1998/1999). Como indica VAliENTE, los planes son relativamente fáciles de aprobar por el pleno municipal, y muy difíciles de ejecutar, debido a 'la indiferencia, cuando no a la oposición de las restantes autoridades municipales', entre las cuales, en ocasiones, hay que incluir las concejalías y áreas de servicios sociales que, en lugar de ser aliados, se comportan como competidores (VAliente, 1998/1999: 182).

En cuanto al contenido de los planes de igualdad, existe una gran variedad de ámbitos. Destacables son los campos de la formación ocupacional y el empleo, violencia, participación, sensibilización, salud, cultura, educación, servicios sociales, atención y asesoramiento jurídico y psicológico, urbanismo.

Según el estudio de SAMPEDro (1992), el tipo de intervención local en el fomento de la igualdad de género depende en gran medida del tamaño del municipio. Los municipios de mayor entidad tienen por lo general programas de igualdad integrales y estables, específicamente dirigidos a mujeres. Mientras que en municipios de menor tamaño (es decir menores de 20.000 habitantes) se hacen actividades más puntuales (8 de marzo), o no diseñadas según el objetivo de la igualdad de género, y se atienden casos individuales de especial gravedad.

En general, en municipios mayores de 20.000 habitantes, existen programas estables de atención a la mujer y unas estructuras mínimas de atención específica a la mujer, con un personal determinado para esta tarea (normalmente una psicóloga, una abogada y una asistente social). Los Ayuntamientos mayores de 50.000 habitantes se ocupan también de la promoción laboral de la mujer, atención específica a mujer (malos tratos, etc.), casas de acogida, asesoría jurídica gratuita en colaboración con los Colegios de Abogados, formación de policía local en casos de malos tratos a mujeres, coeducación.

\section{Valoración de las políticas de igualdad municipales}

¿Que valoración ofrecen de las políticas de igualdad locales los dos estudios señalados? VALIENTE considera que, si bien por 
un lado los organismos de igualdad locales no representan una variedad institucional en sí, por otro lado tienen unas características específicas que las distinguen tanto de las instituciones de igualdad centrales como de las autonómicas:

1) el hecho de prestar servicios directamente a la población obliga a las administraciones municipales a tener en cuenta sus preferencias, aunque sea por razones meramente electorales;

2) la proximidad entre actores institucionales y ciudadanía facilita la inclusión de las asociaciones de mujeres en la elaboración de las políticas de igualdad de género locales (VALIENTE, 1999).

Estos rasgos de comunicación con la ciudadanía e inclusión de las preferencias de mujeres por parte de las administraciones locales llevan a la autora a dar una valoración moderadamente positiva de las políticas de género locales. Esta valoración incluye también el contenido de las actuaciones, aunque se critica el hecho de que el objetivo de algunas actividades no sea tanto el fomento de la igualdad de género, sino el entretenimiento, a veces incluso a través de iniciativas de carácter abiertamente tradicional (como talleres de corte y confección o cursos de maquillaje). Cualquier forma de política para mujeres, según VALIENTE, tiene la ventaja de dar 'legitimidad política a la cuestión de la igualdad entre los géneros como un asunto al que las autoridades deben prestar cierta atención y dedicar recursos y esfuerzos' (VALIENTE, 1998/1999: 186).

El estudio de SAMPEDRO (1992) lamenta la falta de una verdadera política integral de la mujer en el ámbito local. Se critica la existencia de planteamientos de tipo 'asistencial' y puntual, en lugar de actuaciones globales y de programas estables, y la limitación de las políticas de igualdad locales a municipios de mayor tamaño. Además, se destaca la poca coordinación tanto en el ámbito intramunicipal, como entre organismos de igualdad locales, autonómicos y centrales. Quizás algunos de los problemas detectados por SAMPEDRO a principio de los noventa se hayan ido solucionando en los últimos años. Sin embargo, los objetivos que plantea esta ponencia siguen siendo importantes para unas políticas de género eficaces en el conseguimiento de la igualdad y merece la pena tenerlos en cuenta cuando se elaboran planes y programas para la mujer. También cabe destacar que, si bien se tiene bastante asumida la necesidad de elaborar políticas integrales para la mujer, en la práctica de trabajo la horizontalidad y la coordinación interdepartamental entre distintos niveles institucionales está aún lejos de conseguirse. Esto limita la oferta de servicios de igualdad para los municipios de menor tamaño. Las indicaciones de SAMPEDRO siguen siendo, por esta razón, indicaciones útiles a las que prestar atención.

\section{Políticas de igualdad en el nivel local: los Ayuntamientos de la provincia de Barcelona}

Una vez introducido el marco legal de las políticas de género locales y algunos elementos característicos de estas actuaciones municipales, voy a detenerme sobre las políticas de género en los Ayuntamientos de la provincia de Barcelona. Este análisis tiene en cuenta los dos estudios señalados de SAMPEDRO y VALIENTE 6 y se funda también en un trabajo empírico mediante entrevistas telefónicas 7 a nueve Ayuntamientos de la provincia de Barcelona. El tamaño de los Ayuntamientos considerados varia entre 27.000 y 185.000 habitantes ${ }^{8}$, pero en cualquier caso son municipios con una población mayor de 20.000 . Esto significa que pueden contar con concejalías de la mujer, regidoríes de la dona, programas o planes de igualdad, servicios de atención a la mujer, y plantilla variable, que incluye, por lo general, una técnica, una asistente social, una abogada y una psicóloga.

\section{Origen de las regidoríes de la dona}

La razón que explica la emergencia de políticas de igualdad locales en los Ayuntamientos de Barcelona es más política que legal, pues depende sobre todo de la voluntad política de una persona o de un partido ${ }^{9}$. La segunda explicación de la intervención municipal en materia de igualdad se halla en las demandas sociales de asociaciones de mujeres y profesionales ${ }^{10}$. Finalmente, hay quien ofrece una explicación mixta del surgir de políticas de género locales, es decir, por efecto tanto de la voluntad política como de la demanda social ${ }^{11}$.

En el caso de los Ayuntamientos considerados, la orientación ideológica del partido en el gobierno podría tener una cierta relevancia para explicar el desarrollo de políticas locales de igualdad, ya que en 6 de los 9 municipios estudiados el PSC (Partit dels Socialistes de Catalunya) estaba en el gobierno cuando se creó la regidoría ${ }^{12}$.

Sin embargo, este factor no es necesariamente determinante, por tres razones:

1) no todos los Ayuntamientos PSC tienen regidoría;

2) en dos Ayuntamientos donde se afirma que la regidoría se creó por voluntad política, los partidos al gobierno son Convergencia i Unió ${ }^{13}$ e Iniciativa per Catalunya ${ }^{14}$; 
3) el número de Ayuntamientos considerados es excesivamente limitado como para ser significativo en términos de análisis cuantitativo.

Más que el color del partido, parece ser un factor determinante la voluntad política personal de la concejala que decidió iniciar políticas de igualdad a través de una regidoría.

\section{Los factores explicativos de las políticas de igualdad en los Ayuntamientos de Barcelona}

Entre las razones más generales del porqué se empiezan a hacer políticas de igualdad en el ámbito municipal de la provincia de Barcelona, si ya existen en los niveles autonómico y central, hay tres que merece la pena destacar en este estudio, porque cada una ofrece una perspectiva interpretativa distinta:

1) La primera razón es la más evidente, es decir, el municipio es la institución más cercana a la ciudadanía y la que tiene incidencia directa sobre la población ${ }^{15}$. Desde el nivel municipal es más fácil detectar las necesidades reales de la ciudadanía (por ejemplo, a través de los servicios de atención a la mujer se detecta que necesidades existen en distintos sectores de la población de mujeres) y, a partir de allí, se pueden elaborar las políticas de igualdad y las acciones concretas a medida de las demandas existentes.

2) La segunda explicación se centra en la necesidad de elaborar políticas con un enfoque más global que la atención a mujeres que se podía desarrollar desde el área de servicios sociales, y es la que se podría colocar en la politización del ámbito local que caracteriza el fenómeno del 'nuevo localismo' ${ }^{16}$. Se empezaron a hacer políticas de igualdad locales desde la regidoría para superar el tipo de atención a mujeres que solo consistía en el amparo y para tener un enfoque político orientado a mejorar la situación global de las mujeres. En esta misma dirección se dirige la decisión de implantar planes transversales para la mujer, porqué se vio que los planes específicos solo llegaban a determinadas mujeres y no a todas ${ }^{17}$.

3) La tercera explicación está caracterizada por un mayor realismo político: 'Se hace por presión Europea y acuerdos globales, porque es políticamente correcto hacerlo, no por concienciación. La confirmación de esto es el presupuesto mínimo que se dedica a políticas de igualdad (es poco y está compartido con muchos departamentos): esto es el reflejo del poco interés que hay hacia estas políticas' ${ }^{18}$.

Una nota común, de hecho, es el presupuesto insuficiente que se dedica a las políticas de igualdad y la valoración de que serían necesarios más recursos para poder implementar las medidas políticas de forma satisfactoria. Debido al presupuesto limitado del que disponen, el equipo técnico y las responsables políticas inten- tan, por un lado, convencer a los demás departamentos de que utilicen sus presupuestos para llevar a cabo políticas de igualdad y, por el otro, aprovecharse de los programas y proyectos europeos que les permitan desarrollar iniciativas para la igualdad.

\section{Características de las regidoríes de la dona de los municipios de Barcelona}

La mayoría de las regidories en los Ayuntamientos de la provincia de Barcelona se crearon en la década de los noventa ${ }^{19}$, confirmando la pauta ya encontrada en los municipios de Madrid (Valiente, 1998/1999). Dos Ayuntamientos ya empezaron a desarrollar políticas de igualdad en los años ochenta, pero en estos no se creó concejalía de la mujer ${ }^{20}$. En todos los Ayuntamientos analizados existen formas de participación de la población femenina en el proceso de toma de decisiones, que es uno de los criterios que VALIENTE considera clave para valorar positivamente las políticas de igualdad locales (VALiENTE, 1998/1999).

Las preferencias y demandas de las mujeres se recogen, en muchos de los casos, a través de Consells u órganos de participación de carácter consultivo compuestos normalmente por todas las entidades sociales, es decir asociaciones de mujeres y de carácter socio-cultural, partidos y sindicatos a través de su secretaría de la mujer, mujeres que sin ser asociadas tienen interés y una cierta competencia en materia de género. En algunos casos, los Consells trabajan mediante mesas de trabajo, como en Sabadell, donde existen 4 mesas: mujer y mundo laboral, violencia y malos tratos, sensibilización, recuperación y memoria histórica de mujeres de Sabadell.

La impresión es que el asociacionismo feminista o de mujeres preexistente a la creación de políticas de igualdad locales no estuviera excesivamente desarrollado en el ámbito municipal, como demuestra también el hecho de que ninguna de las entrevistadas hizo referencia al movimiento de mujeres como causa del establecimiento de políticas de igualdad locales.

\section{Los planes de igualdad en los municipios de Barcelona}

La casi totalidad de los municipios considerados trabajan a través de planes de igualdad, que, aunque abarcan una gran diversidad de medidas adoptadas, comparten algunos temas comunes como son la atención directa, la sensibilización, la formación, la prevención de la violencia en contra de las mujeres, y la educación.

En algunos casos, como el de Granollers, el plan municipal incluye tanto temas específicos del Ayuntamiento como temas 
comunes que se trabajan juntamente en el Consejo Comarcal. Este aglutina municipios con mas de 20.000 habitantes y trabaja para asistir a mujeres que viven en municipios menores que tienen menos posibilidades de atender a las necesidades de su población femenina.

En Sant Boi de Llobregat se intenta trabajar la transversalidad a través de diferentes programas cuya tarea es la de poner en actuación las medidas contenidas en el plan municipal21. Dentro de cada proyecto se desarrollan los ejes del plan: sensibilización sobre la igualdad; concienciación sobre la violencia contra las mujeres; formación; participación activa con el Consell y las Comisiones de trabajo.

Determinados Ayuntamientos, como Vilafranca del Penedès, tienen una regidoría solo para el plan de igualdad en lugar de una regidoría de la dona; por lo tanto, todas las actividades del organismo de igualdad se dirigen a la realización de los objetivos del plan (éstos son: garantizar los derechos fundamentales desde la perspectiva de género, favorecer las relaciones interculturales entre las personas que provienen de otros países, ofrecer formación, potenciar la participación en temas de interés colectivo desde la perspectiva de género).

Otras concejalías de la mujer se centran en temas específicos: la formación es un eje fundamental de la política de igualdad de Vilanova i la Geltrú. Para ello se organizan cursos de formación para la policía local específicos para el tema de malos tratos a mujeres (juntamente a la asociación Tamaia, especialista en formación en esta materia) y se forman en temáticas de género las psicólogas que trabajan en la asesoría psicológica, debido a que la Universidad no provee estos contenidos educativos. En definitiva, dentro del marco común de la lucha a la discriminación de género por parte de los municipios barcelonenses, existe una gran variedad de soluciones para la articulación de los planes de igualdad.

\section{Centros de atención directa a mujeres en los Ayuntamientos de Barcelona}

Un aspecto de las políticas de igualdad que es común a los Ayuntamientos de Barcelona estudiados consiste en la creación y mantenimiento de centros de atención directa a mujeres. La fundación de estos centros es contemporánea o algo posterior al establecimiento de las concejalías, es decir entre los noventa y los primeros años del 2000, y es uno de los puntos contemplados en los planes de igualdad 22 .

Los centros de atención directa, ofrecen una atención individualizada a todas las mujeres que acudan a ellos, proporcionando unos servicios de información y asesoramiento de tipo jurídico, psicológico, laboral, en algún caso también terapéutico. Según un informe del Ministerio de Asuntos Sociales (1992), los primeros centros en ofrecer una asistencia más directa de este tipo han sido los CIDEM o centros de información sobre los derechos de la mujer. Los CIDEM fueron creados, a partir del 1982, con el objetivo de 'fomentar la prestación de servicios en favor de la mujer y en particular, los dirigidos a aquellas que tengan una especial necesidad de ayuda, y de facilitar información a las mujeres sobre los derechos que la Constitución y el ordenamiento jurídico les reconocen, así como los medios para hacerlos' (Ministerio de Asuntos Sociales, 1992: 1). La mayoría de los CIDEM están gestionados por el Instituto de la Mujer en conjunto con los Ayuntamientos o tan solo por los Ayuntamientos; algunos están dirigidos por el IM solamente; otros están gestionados por las Diputaciones.

Desde la década de los 80 , el número de centros de información de derechos de la mujer, así como de casas de acogida y centros para el embarazo (de adolescentes) ha crecido de forma consistente $^{23}$, evidenciando la existencia de una demanda de servicios personalizados por parte de las mujeres. La demanda es por lo tanto uno de los factores explicativos del origen y de la difusión de la atención directa en los Ayuntamientos ${ }^{24}$.

Por lo que concierne el perfil de las usuarias, tanto en el informe ministerial como en el estudio de SAMPEDRO se destaca que la mayoría de las consultas que se reciben en los centros de información solicitan asesoramiento jurídico para una posible separación o divorcio, solicitando a la vez un puesto de trabajo. Otras demandas de las usuarias conciernen malos tratos y problemas familiares; educación sexual y planificación familiar; información sobre servicios y recursos sociales (SAMPEDRO, 1992; Ministerio de Asuntos Sociales, 1992).

Aparte de los centros de información y asesoramiento sobre derechos de la mujer, la atención directa se concretiza también en servicios de varios tipos como por ejemplo: casas de acogida a mujeres en situaciones de especial dificultad, mujeres maltratadas, madres solteras, prostitutas, toxicómanas, ex-reclusas; asistencia especializada a mujeres víctimas de violencia en el ámbito de la Policía y la Guardia civil; información telefónica gratuita para ofrecer a las mujeres información y asesoramiento puntual acerca de sus derechos y de los recursos sociales existentes. 


\section{Profesionales de los centros de atención}

El grado de equipamiento y plantilla de estos centros varía mucho, pero en general, suele consistir de una abogada y/o una psicóloga y/o una asistente social, normalmente con un horario de atención no continuado. Algunos municipios disponen también de una terapeuta (Sabadell, Barberà del Vallès) o de una pedagoga (Casaldona de Vilafranca del Penedès). Los Ayuntamientos de mayor tamaño suelen disponer de una plantilla más numerosa y con contrato laboral del mismo Ayuntamiento (Sabadell). También se recurre a personal contratado por otras entidades que dedica un cierto numero de horas a servicios de atención directa a mujeres. En ciertos casos las profesionales son compartidas entre distintos departamentos (salud, servicios sociales).

La inestabilidad laboral y la falta de formación en materia de género del personal técnico (y político), son obstáculos materiales a la consolidación de las políticas de género. Por lo tanto, los estudios sobre políticas locales de género (SAMPEDRO, 1992; VALIENTE, 1998/1999) subrayan la necesidad de personal cualificado para consolidar programas locales de promoción para las mujeres. La formación en materia de igualdad de género del personal tanto técnico como político es fundamental para llevar a cabo un trabajo eficaz de atención a la mujer.

\section{Oferta de servicios y sujetos beneficiarios de la atención directa}

En los centros de atención directa se atiende generalmente a toda mujer que sufra discriminación por razón de sexo. Este objetivo, que está contemplado en los planes de igualdad, se justifica por la idea de que la forma más eficaz para ayudar a las victimas de discriminación de género es con un servicio específico personalizado. Los servicios que se ofrecen suelen consistir en información general a mujer; asesoría jurídica y psicológica (para la mujer y, a veces, para niñas y niños que hayan tenido experiencias de violencia doméstica), en algún caso también laboral (información y orientación); asistencia social y terapéutica.

Si bien la atención jurídica, social y psicológica está suficientemente establecida en los centros, los servicios de asesoría laboral no están tan difundidos, a pesar del papel fundamental que tiene la independencia económica de la mujer tanto para la mejora de sus condiciones materiales de vida como para el desarrollo de una capacidad de elección autónoma de sus posibilidades de vida. En diversos municipios europeos se ofrecen una serie de servicios de asesoría laboral para mujeres como bancos de datos, ayuda personalizada en la búsqueda de trabajo y ofertas formativas, ayuda para preparar curriculum y difusión de los mismos en las empresas, encuentros informativos individuales y grupales, sensibilización de las empresas locales a los proyectos y a la nor- mativa nacional y europea que favorece la asunción de mujeres, cursos de formación en los puestos de trabajo para concienciar a las trabajadoras de sus derechos laborales 25 .

Con respecto a la extensión de servicios a la familia, existen Ayuntamientos que ofrecen atención a niñas y niños que han sufrido experiencias de violencia domestica o abusos, tanto con asistencia psico-pedagógica como a través de actividades de coeducación a la igualdad.

Además, considero importante que en las intervenciones se intente mejorar el nivel de concienciación de las mujeres, a través del conocimiento de sus propios derechos y de formación al pensamiento feminista, y acrecer la autoestima de las mujeres, para que se encuentren más preparadas a reaccionar a las posibles discriminaciones y explotaciones a las que se tengan que enfrentar. Entre las recetas ya existentes a este respecto se pueden destacar: los Centros de Recursos Locales para mujeres en Suecia, que son centros de formación, concienciación e información para las mujeres, que incluyen seminarios, cursos y actividades de formación ${ }^{26}$; el servicio de información itinerante de la Federación Mujeres Progresistas sobre derechos de la mujer, derechos reproductivos, laborales, asistencia social, y que se ha desplazado en municipios rurales de Madrid para difundir información a mujeres donde es más difícil recibirla 27; los study circles suecos, que dan formación para mujeres desempleadas para motivarlas y animarlas en la búsqueda de trabajo y en fortalecer su autoestima ${ }^{28}$.

En algunos centros se ha planteado la cuestión relativa a la posibilidad de ofrecer servicios a los hombres maltratadores. Esto depende de la filosofía que se adopta en el centro de atención, de sus recursos, y de las finalidades de la asistencia que se ofrece. Evidentemente las medidas disciplinares son útiles pero al mismo tiempo deberían representar solo una parte del tratamiento reservado a los maltratadores. El otro aspecto de una intervención que quiera ser preventiva de futuras violencias sexistas es la reinserción del reo a través de una educación a la igualdad de género y a la no-violencia y de un apoyo psicológico y terapéutico. Para esto se requieren centros especializados en reinserción que puedan contar con presupuestos y equipos específicos.

Uno de los problemas que se plantean los centros de atención que quieran trabajar con maltratadores es la escasez de recursos económicos de los organismos de igualdad locales y la necesidad de que los políticos asuman su responsabilidad con respecto a esta cuestión. Si los centros de atención a mujer ofrecieran servicios a los maltratadores se harían cargo del trabajo de reinserción para cuya solución las instituciones tienen que tomar responsabilidad y establecer medidas ad hoc como la creación de centros especializados, la inversión de presupuestos específicos y la difusión de una cultura de la igualdad en todos los ámbitos, desde la educación escolar hasta la comunicación mediática. La asunción del trabajo de reinserción de maltratadores por parte de los cen- 
tros de atención a mujer no solo privaría a las mujeres de un presupuesto que ya es insuficiente para sus propias necesidades, sino que representaría un desagravio de responsabilidad para las instituciones competentes que, utilizando el trabajo de las profesionales de los centros de mujer, no tomaría ulteriores medidas en este asunto. Además, la intervención de los centros de atención a mujer dirigidos a hombres maltratadores sería más bien de carácter paliativo, debido a la carga de trabajo que ya tienen las profesionales y el presupuesto limitado del que disponen. Tal vez sería más útil que las profesionales de los centros de atención a mujer presionaran a las instituciones para que se abrieran centros de reinserción de maltratadores y se fortaleciera la educación y formación a la igualdad de género en todos los ámbitos sociales.

Una receta de intervención preventiva de la violencia domestica para los centros de atención que quieran trabajar con maltratadores es lo que se ha realizado en Helsinki con el programa TRAINING $^{29}$, con el que se involucra el hombre violento en un proceso formativo-terapeutico desde la primera señal de violencia recibida por parte de policía, escuelas, médicos, etc. (esto requiere una sinergia entre los distintos actores sociales). Los profesionales (asistentes sociales, psicólogos, terapeutas) contactan el violento y lo convencen a empezar una terapia que consta de dos fases: la primera, de uno a seis meses, de terapia individual, dirigida a concienciar la persona de su actitud violenta y de los daños que esto conlleva para su familia; la segunda fase, de unos cuatro meses, de encuentros colectivos con grupos de seis/ocho personas con el mismo problema, en los que los profesionales trabajan para remover las causas de la violencia.

\section{Mainstreaming de género en los municipios de Barcelona}

Desde la introducción del mainstreaming de género o transversalidad por parte de las Naciones Unidas antes y de la Unión Europea después, este concepto ha entrado a formar parte de la política nacional, o por lo menos de su diccionario. Sin embargo, hasta la fecha la tendencia en los países europeos ha sido la de adoptar un enfoque más 'integracionista' a la transversalidad, que pretende abordar los asuntos de género dentro del paradigma político existente, en lugar de un enfoque 'cambio de agenda' (agenda-setting), que implica la transformación y la redirección de la misma agenda política (JAHAN, 1995). El enfoque 'cambio de agenda' requiere transformaciones en las estructuras y en los procesos de toma de decisión, en la articulación de los objetivos, en la prioritización de los temas de géneros entre otros asuntos relevantes, y en general un replanteamiento de las configuraciones institucionales que apoyan la presente división del poder en la toma de decisiones (JAHAN, 1995).

¿Cómo se aplica la transversalidad en las políticas locales de los Ayuntamientos de Barcelona? ¿Qué dificultades y obstáculos existen para poner en marcha la transversalidad? ¿Qué estrategias y buenas prácticas se han desarrollado para ello?

La primera observación relativa a la aplicación del mainstreaming de género en los municipios de Barcelona es que, más que de transversalidad se puede hablar de coordinación interdepartamental, como también destacan las personas entrevistadas ${ }^{30}$. Se ha conseguido que el personal de distintos departamentos empiece a trabajar conjuntamente para conseguir unos objetivos comunes de igualdad.

En algunos casos ${ }^{31}$ la coordinación interdepartamental es uno de los puntos del programa de igualdad de la regidoría; en otros casos 32 el mismo programa pretende ser 'transversal' (o más bien coordinado) en el sentido de comprender en un mismo plan todos los proyectos (cultura, infancia, servicios sociales, etc.), de forma que para llevarlos a cabo se requiera, necesariamente, una coordinación entre los departamentos. En ambos casos, el enfoque sigue siendo integrador más que de 'cambio de agenda', pero puede representar un inicio de transversalidad si consigue evolucionar hacia la transformación de la agenda política (estableciendo prioridades y objetivos) y de las estructuras de toma de decisiones (desarrollando la dimensión de participación y horizontalidad del trabajo).

Para determinados temas parece más fácil trabajar de forma coordinada. Es el caso de la violencia hacia las mujeres, que, beneficiándose del actual interés mediático por el tema, permite implicar a diversos departamentos, como policía, servicios sociales, o salud ${ }^{33}$. Sin embargo, para temas que no disfrutan del mismo 'atractivo' mediático la coordinación se traduce en un esfuerzo de sensibilización de otros departamentos hacia la igualdad de género, y no siempre con el éxito deseado.

Existen varias formas de gestionar la 'transversalidad'. Unos municipios ${ }^{34}$ organizan comisiones de trabajo (salud y mujer; servicios sociales y mujer, policía local y mujer, etc.) que permitan coordinar estrategias conjuntas de actuación. Otros 35 han suprimido las comisiones de trabajo formadas por un/a representante de cada departamento (y donde se discutían los temas de cada departamento), para pasar a formas de trabajo en las que la regidoría trata directamente con los responsables de cada departamento. En ciertos casos ${ }^{36}$, todas las áreas departamentales se juntan para elaborar el plan, aunque se lamenta que a la hora de ponerlo en práctica se ponga en evidencia la diferencia entre las actuaciones de técnicos más y menos sensibilizados al tema de la igualdad.

\section{Obstáculos a la transversalidad}

La dificultad en la aplicación de la 'transversalidad' es la queja principal que comparten las personas que trabajan en políticas de género municipales. Se pueden distinguir cinco tipos de obstácu- 
los, tanto ideológicos como estructurales, a la coordinación interdepartamental:

La estructura burocrática del trabajo institucional está caracterizada por la parcelación.

2 Hay poca costumbre de trabajar en equipo y de forma coordinada, debido también a una cierta falta de cultura cooperativa.

3 La cultura de trabajo existente es jerárquica, es decir vertical, mientras que la transversalidad requiere que todo el mundo esté al mismo nivel, es decir, horizontalidad.

4 La sobrecarga de trabajo en cada área dificulta el trabajo coordinado, que, al menos al principio, implica un aumento de trabajo.

5 Existe una escasa concienciación sobre el tema de la igualdad de género, e incluso resistencia a trabajar en asuntos de igualdad debido a prejuicios culturales sexistas, tanto por parte de responsables técnicos como políticos.

Los obstáculos a la puesta en práctica de la coordinación interdepartamental demuestran que una verdadera aplicación de la transversalidad requiere cambios sustanciales en la estructura del trabajo institucional y burocrático, en las jerarquías de poder, y en la mentalidad y la postura hacia la igualdad de género. La puesta en juego de la transversalidad es una revolución en las relaciones humanas y laborales que aún no ha sido suficientemente asumida.

\section{Recetas locales para la transversalidad}

Existe una gran variedad de recetas para introducir la transversalidad en las políticas de género locales. Una antigua y eficaz receta consiste en dar formación para favorecer el cambio de mentalidad y de actitud. Un pueblo de Valencia, beneficiándose de un programa europeo, consiguió dar formación en materias de transversalidad y de igualdad de genero a todo el personal municipal, tanto político como técnico ${ }^{37}$. La Diputación de Barcelona también promueve y ofrece formación en transversalidad. De los países europeos, Suecia ha sido hasta ahora el único que ha formado a todo su personal gubernamental -ministros incluidos- en materia de mainstreaming de género, mientras que Bélgica realiza cursos de formación a las acciones positivas dirigidos a todas las administraciones públicas ${ }^{38}$. Una de las indicaciones de estas experiencias es que, para alcanzar una puesta en práctica de forma efectiva, la formación en transversalidad e igualdad de género sea obligatoria ${ }^{39}$ y comprenda a todo el personal municipal ${ }^{40}$, desde los profesionales a los políticos. De esta forma, en principio, las medidas de igualdad no tendrían que depender de la voluntad política y técnica que se encuentre.
Entre las recetas prácticas cabe destacar la propuesta de que cada área tenga un porcentaje de presupuesto para la igualdad de género $^{41}$. Con ella, se intenta solventar el problema de la falta de recursos presupuestarios propios del plan transversal, asumiéndolos cada departamento. Debido a que los cambios de mentalidad $\mathrm{y}$ de actitud hacia la igualdad requieren un cierto tiempo para asentarse, la propuesta de una autonomía presupuestaria para género en cada área podría garantizar, mientras tanto, la posibilidad de llevar a cabo iniciativas para la igualdad en un ambiente aún en fase de formación.

La multiplicación de contactos y seminarios entre entidades municipales de distintos países europeos es de gran utilidad tanto para poder intercambiar experiencias, ideas, y tácticas políticas como para dar legitimidad al esfuerzo de todas las profesionales que trabajan en la igualdad ${ }^{42}$. El contacto con las instituciones de igualdad de género de la Unión Europea y ONGs europeas de mujeres, como el Lobby europeo de mujeres ${ }^{43}$, también es muy útil para acceder a información actualizada sobre normativa, programas, recursos y estrategias de género. En el nivel local, puede ser interesante trabajar a la construcción de redes (Consell comarcal provincial) para la obtención de recursos y el comparto de experiencias e iniciativas.

Lo importante es no resignarse a permanecer en la fase integracionista' de adopción del mainstreaming, y seguir trabajando para que la transversalidad sea una verdadera revolución en la mentalidad y en la práctica política. Para esto, es fundamental enfocar bien los objetivos políticos que se quieran conseguir y elaborar las estrategias más eficaces para obtenerlos.

\section{Conclusiones: límites de las políticas de género locales y retos para el futuro}

Entre los límites de la intervención local en materia de igualdad, Sampedro destaca el hecho de que 'se prima, en general, la atención individual frente a la intervención comunitaria, las acciones reparadoras frente a las preventivas'. Un ejemplo es 'el desigual desarrollo de actuaciones dirigidas a mujeres maltratadas, madres solteras, mujeres sin recursos económicos con hijos a su cargo, etc., y las relacionadas con aspectos tan cruciales para la prevención de la desigualdad como la formación profesional y el empleo, la coeducación, etc.' (SAMPEDRO, 1992: 38).

De esta afirmación se puede deducir que el objetivo hacia el cual tienen que trabajar las políticas de género locales es la articulación de actuaciones globales dirigidas a prevenir las discriminaciones más que a curarlas. La atención directa, por ejemplo, para tener en cuenta este objetivo global y preventivo, debería superar un enfoque de tipo asistencialista y dirigir los esfuerzos hacia el desarrollo de mujeres autónomas, conscientes de sus derechos y del papel que tanto hombres como mujeres deberían 
desempeñar para conseguir la igualdad. Esto implica también la promoción de una sociedad aún más concienciada sobre el tema de la igualdad entre mujeres y hombres, a la que se puede llegar solamente invirtiendo en educación y formación en todos los niveles de edad y en cada grupo social. Ambos elementos son imprescindibles para cualquier política de igualdad que aspire a generar cambios profundos en la mentalidad y en las pautas de comportamiento de las personas, capaces de recoger el patrimonio ideológico del feminismo, institucional y no institucional.

Un aspecto de las políticas de género que es importante destacar es que éstas son el resultado de un trabajo en red entre organismos de carácter supra-, inter-, sub- y nacionales. Los distintos niveles de actuación se complementan recíprocamente para articular medidas políticas más eficaces para el avance de la igualdad de género. En este proceso tienen un papel significativo tanto la red de mujeres, que permite la coordinación cotidiana de los esfuerzos individuales, como los foros mundiales, europeos, nacionales y locales, que permiten compartir pensamientos y estrategias políticas.

La lógica local requiere que las intervenciones se adapten a los problemas específicos y a las necesidades de la población (Equipo de Análisis Política, 2000). Sin embargo, para poder mejorar sus políticas e innovar sus estrategias de acción, los organismos de igualdad locales no deben perder de vista la visión global del problema y deben poderse beneficiar de las perspectivas sobre género que se desarrollan desde distintos foros de mujeres. El eslogan 'piensa global, actúa localmente' ('think global, act local), en su simplicidad callejera, es capaz de expresar uno de los retos de nuestra época de globalización con claridad e inmediatez. La falta de contacto entre las distintas ramas que trabajan hacia la igualdad implicaría una perdida de las ventajas de la descentralización, pues lo local sin lo global empobrecería su propia visión. Es cierto también que para mantener una perspectiva abierta hacia lo global es fundamental un empleo eficaz de la red.

Según el equipo de análisis de políticas públicas de la Universidad Autónoma de Barcelona (2000) el principal reto de las políticas locales es precisamente la gestión de la red y de las relaciones intergubernamentales. Los estudiosos afirman que la elaboración de políticas locales estratégicas es imposible desde una perspectiva de actuación solitaria, y es posible solo a partir de pautas de trabajo relacionales que extiendan el diálogo con un elevado numero de actores institucionales y no institucionales. Para ello, hay que superar los obstáculos puestos tanto por las delimitaciones institucionales como por la resistencia humana al cambio de las pautas de trabajo y de relación. El apoyo de la red, además, es especialmente importante para dar legitimidad a las reivindicaciones de las mujeres en todos los lugares sociales e institucionales.

Pensar globalmente no quiere decir solamente trabajar en red, sino también tener un proyecto general y unos objetivos cla- ros sobre el tipo de igualdad de género que se aspira a conseguir. Las respuestas de las administraciones municipales a las demandas de las mujeres deben estar enfocadas desde una determinada idea de igualdad de género, a partir de la cual se definen los objetivos a corto, medio, y largo plazo. El análisis de VALIENTE resalta este aspecto, cuando valora positivamente los organismos de igualdad locales que sepan ligar los 'intereses prácticos de género' (los que 'reflejan las necesidades inmediatas de distintos grupos de mujeres, tal como ellas mismas las definen') con los intereses 'estratégicos' de género (los que 'se elaboran a partir del análisis de la condición de las mujeres en un momento dado, y de su comparación con una situación (ideal) más igualitaria'). (VALIENTE, 1998/1999: 184)

En definitiva, la forma en la que se enmarcan las medidas políticas de igualdad es determinante para el impacto que tienen sobre las condiciones de vida de las mujeres y el progreso de la igualdad. El enfoque de las intervenciones en los centros de atención directa, por ejemplo, puede o bien favorecer la independencia y participación de la mujer en la vida pública, y estimular el reparto de las responsabilidades entre los sexos, o bien mantener el papel tradicional de la mujer, crear dependencias a través de intervenciones de tipo asistencialista, y no cuestionar el papel del hombre en una sociedad más igualitaria.

Otro reto fundamental de las políticas de género locales es dar más espacio a la participación de las mujeres, que debería ser más fácil conseguir gracias a la proximidad de las instituciones municipales con la ciudadanía. Tanto SAMPEDRO como VALIENTE destacan la importancia de diseñar medidas de igualdad locales a partir del conocimiento de las necesidades y demandas de los colectivos femeninos. VALIENTE nos informa de las distintas prácticas en uso en la Comunidad de Madrid para incluir las preferencias de las mujeres, entre ellas cuestionarios a las mujeres de la ciudad sobre sus expectativas/demandas, convocatorias públicas, y participación directa de asociaciones de mujeres en la articulación de las medidas políticas.

Muchos de los municipios de la provincia de Barcelona tienen Consells u órganos de participación ciudadana específicos de mujeres, Consells de dones, o bien abiertos a grupos de mujeres, partidos políticos, sindicatos, y expertas. Es necesario seguir estimulando desde las instituciones locales la participación de grupos de mujeres, en particular los que tienen un enfoque más claramente reivindicativo y feminista, debido a las propuestas de transformación que puedan aportar (SENSAT y VARELLA, 1998). La ventaja que tienen las administraciones municipales sobre los otros niveles institucionales es, como se ha dicho, la proximidad con la ciudadanía. Tal vez el reto menos evidente pero no por esta razón menos significativo al que las políticas de género locales tienen que enfrentarse es una gestión de esta proximidad que sepa valorar y dar voz al potencial humano de los distintos grupos sociales que componen una determinada comunidad local. 


\section{* Agradecimientos}

Este trabajo de investigación ha sido financiado por la Secretaría de Estado de Educación y Universidades. Mis agradecimientos van también al Ayuntamiento de Sabadell y a la Diputación de Barcelona por invitarme a participar en las Jornadas sobre "Centros municipales de atención a la mujer" que han sido al origen de este estudio. Deseo agradecer además a todas las personas que con sus observaciones y colaboración me han ayudado a conocer mejor los organismos de la mujer de la provincia de Barcelona y, al mismo tiempo, me han permitido compartir un interés común acerca del progreso de la igualdad de género. Por lo tanto, muchas gracias a Joana Aloy, Rosa Botella, Marisol Caro, Rosa Catalá, Maite Forné, Mariona Junyent, Cristina Moga, Sonia Ruiz, Nuria Suarez y Roser Puig.

** Universidad de Zaragoza

1 Para una explicación del concepto de mainstreaming véase la sección 2 'El mainstreaming de género en los niveles central y autonómico'.

2 Por lo que concierne a Cataluña, el traspaso de funciones desde el nivel central al autonómico se da con las siguientes leyes: Real Decreto 352/81 de 18/9; Ley de Servicios Sociales 26/85 de 27/12 art. 5.2f; mientras que los órganos competentes son establecidos por la Ley 11/89 que crea el Institut Català de la Dona adscrito al Departamento de Presidencia.

3 Entrevista a Pilar Perez Cantó, Directora del Instituto Universitario de Estudios de la Mujer, Universidad Autónoma de Madrid, 18/11/99.

4 La traducción de mainstreaming con 'transversalidad' es incompleta debido a que no traduce la idea de introducir la perspectiva de género en la corriente dominante, sino que refleja una parte del concepto de mainstreaming que es la aplicación horizontal de la perspectiva de género en todas las áreas.

5 Un buen ejemplo de las nuevas estrategias integrales en el ámbito municipal es el Plan Integral para la Igualdad de Oportunidades 1998-2002 de la Diputación de Barcelona. El Plan, a partir de un análisis del contexto social de las mujeres, articula unos objetivos bien definidos y las correspondientes líneas estratégicas que hay que seguir; además incluye una evaluación de la implementación de las distintas líneas estratégicas desarrolladas.

${ }^{6}$ Otro estudio sobre los Ayuntamientos catalanes es el de Judith Astelarra, Politiques dels ayuntaments de Catalunya a favor de les dones, Barcelona, Institut Català de la Dona, 1994.

7 Las entrevistas semi-estructuradas fueron realizadas en diciembre de 2002. Incluyeron los siguientes Ayuntamientos de la provincia de Barcelona con sus relativas responsables de igualdad: Barberà del Vallès (Marisol Caro), Granollers (Nuria Suárez), Sabadell (Maite Forné), Sant Adrià de Besòs (Joana Aloy), Sant Boi de Llobregat (Roser Puig), San Cugat del Vallès (Rosa Botella), St Feliu de Llobregat (Cristina Moga), Vilafranca del Penedés (Mariona Junyent), Vilanova i la Geltrú (Rosa Catalá).

${ }^{8}$ El número de habitantes por cada Ayuntamiento considerado es aproximadamente el siguiente: Barberà del Vallès 27.000, Granollers 53.000, Sabadell 185.000, Sant Adrià de Besòs 32.000, Sant Boi de Llobregat 80.000, San Cugat del Vallès 66.000, St Feliu de Llobregat 42.000, Vilafranca del Penedés 32.000, Vilanova i la Geltrú 55.000 .

${ }^{9}$ Entrevistas: Barberà del Vallès, San Cugat del Vallès, Sant Boi de Llobregat, St Feliu de Llobregat, Vilafranca del Penedés, Vilanova i la Geltrú.

10 Entrevistas: Sabadell, Granollers.

11 Entrevista: Sant Adria de Besos.

12 Entrevistas: Granollers, Barberà del Vallès, Sant Adria de Besos, Sant Boi de Llobregat, Vilafranca del Penedés, Vilanova i la Geltrú.

13 Entrevista: Sant Cugat del Vallès.

14 Entrevista: St Feliu de Llobregat.

15 Entrevistas: Sant Cugat del Vallès, Vilafranca del Penedés.

16 Entrevista: Sant Boi de Llobregat.

17 Entrevista: Vilafranca del Penedés.

18 Entrevista: Vilanova i la Geltrú.

19 Sabadell creó su regidoría en 1999, Barberà del Vallès en 1996, Granollers en 19981999, San Cugat del Vallés en 2000, Sant Boi de Llobregat en 1999, St Feliu de Llobregat en 1992, Vilafranca del Penedés en 1996 (hay regidoría del plan).

20 Sant Adrià de Besòs desde el 1983 tiene políticas de igualdad, pero que dependen de servicios sociales; Vilanova y la Geltrú desde 1988-89 desarrolla políticas de 'equidad' a través de una concejalía de servicios personales y un plan transversal.
${ }^{21}$ Los Programas son transversales, es decir, están dirigidos a mujeres inmigrantes, mayores, discapacitadas, etc. Hay una persona que rige cada programa y que coordina las iniciativas con las que rigen los otros programas. De esta forma, la transversalidad se realiza a través de acciones conjuntas entre los diferentes programas.

22 Las fechas de creación de los centros de atención a mujeres en los Ayuntamientos considerados son: 2001 en Sabadell, 2000 en San Cugat del Vallés, Vilanova i la Geltrú tuvo asesoria jurídica desde el 1988 y psicológica desde el 1995, Granollers en 1999, St Adrià de Besòs en 2000, St Boi de Llobregat desde el 1992, St Feliu de Llobregat tiene servicios, en lugar de un centro, de atención desde el 1992, Vilafranca del Penedés tiene un centro de atención directa, Casaldona, desde el 1997, el mismo año de Barberà del Vallès.

23 Comparando los datos relativos al desarrollo de centros de información de derechos de la mujer (CIDEM), casas de acogida, y centros para embarazo (en adolescentes) y nacimientos en 1982 y 1992, se puede comprobar que en 1982 hay 3 centros de información sobre derechos y 0 casas de acogida y centros para embarazo (de adolescentes), en 1992 hay 129 centros de información (dependientes de Ayuntamientos, CCAA, asociaciones), 69 casas de acogida y 641 centros para embarazo, 3 específicos para jóvenes (Ministerio de Asuntos Sociales, 1992). En el último informe estadístico del Instituto de la Mujer, Mujeres en Cifras 2001, se nota que en 1997 había 777 centros de atención y 159 casas de acogida, y que en 2000 estas cifras habían subido a 918 centros de atención y 255 casas de acogida.

${ }^{24}$ En Barberà del Vallès me informan, para confirmar que la atención directa se crea por la demanda, que antes había 50 y ahora hay 100 entrevistas al año en los servicios de atención directa a mujer.

25 Clara Bassanini, Pina Madami, Sportello Donna, Provincia di Lodi (Milano); e-mail: info@pariedispari.it ; Juana López Calero, Secretaría Sectorial de la Mujer, Murcia. Tel.: 00349683666 26 Fax: 0034968366620 E-mail: Juana M.Lopez@carm.es sitio web: www.carm.es/cpre/mujer; Susanna Brodersen FrauenVertreterin im Bezirksamt Steglitz - Berlin, Tel.: 00493079043983.

${ }^{26}$ Ing. Marie Persson CRN (National Resource Centre for Women) Stocolma, Tel.: 004686819100 Fax: 004686816689 E-mail: Ing-marie.persson@nutek.se sitio web: www.nutek.se/nrc

27 Carmen Ortiz, Federación Mujeres Progresistas, Madrid, Tel.: 0034915390238 Fax: 0034915270302 E-mail: fmp@fmujeresprogresistas.com sitio web: www.fmujeresprogresistas.com

28 Janet Eriksson ABF (Arbetarnas Bildningsforbund) Norrkoping (Suecia) Tel.: 004611211300 Fax: 004611211310.

${ }^{29}$ Leena Ruusuvuori, Tel. 00358939672122 fax: 00358939672201 E-mail: leenar@stakes.fi website: www.stakes.fi

30 Entrevistas: Sabadell, San Cugat del Vallès, St Adrià de Besòs, St Feliu de Llobregat 31 Entrevista: Sabadell.

32 Entrevistas: St Boi de Llobregat, St Feliu de Llobregat, Vilafranca del Penedès, Vilanova i la Geltrú.

33 Entrevista: Sant Adrià de Besòs

${ }^{34}$ Entrevista: Barberà del Vallès.

35 Entrevista: Vilafranca del Penedés.

36 Entrevista: Vilanova i la Geltrú.

37 Entrevista: Vilanova i la Geltrú.

38 Ministère de l'Emploi et du Travail- Service Égalitté des chances, Rue Belliard 51, Bruxelles, Tel: 003222334039

39 Entrevista: Barberà del Vallès.

40 Entrevistas: Vilafranca del Penedés; Vilanova i la Geltrú.

${ }^{41}$ La propuesta de la responsable de igualdad de Vilanova i la Geltrú no fue aceptada por el Consejo municipal.

42 Un buen ejemplo ha sido el seminario 'Recetas para el mainstreaming', realizado en Palermo (Italia) por Arcidonna, en el cual se han recogido un gran numero de recetas de buenas practicas para trabajar con el mainstreaming adoptadas en distintos municipios europeos. Véase http://www.arcidonna.it/mainstreaming/Ligth/inglese/Recipes/recipesenl.html

43 Véase http://www.womenlobby.org 


\section{Bibliografia}

AstelarRA, Judith. 1994. Politiques dels ayuntaments de Catalunya a favor de les dones. Barcelona: Institut Català de la Dona.

AstelaRRA, Judith. 1996. "Políticas de igualdad de oportunidades en España y el Reino Unido", en Cristina Brulet Tenas y Pilar CarRasquer Oto (coords), Sociología de las relaciones de género. Madrid: Instituto de la Mujer.

Beveridge, Fiona, Sue NotT y Kylie StePhen. 2000. «Mainstreaming and the engendering of policy-making: a means to an end?», Journal of European Public Policy, Special Issue Women, Power and Public Policy in Europe 7, 3: 385-405.

Bustelo, María. 2002. «Las políticas públicas de igualdad de género en España en los niveles central y autonómico», en Andrés GARCÍA INDA y Emanuela LombARDo (coords), Género y Derechos Humanos. Zaragoza: Mira Editores.

Bustelo, María. 1999. « Políticas Públicas de Igualdad de Género en España: Evolución y Evaluación", en Margarita OrTEGA, Cristina SÁnChEZ y Celia VALIENTE (eds.), Género y Ciudadanía. XII Jornadas de Investigación Interdisciplinarias. Madrid: Instituto Universitario de Estudios de la Mujer.

Equipo de Análisis Política. 2000. "Politicas publicas locales, necesidades y planteamientos emergentes", Universidad Autónoma de Barcelona: 8, Febrero.

GIL RuIZ, Juana María. 1996. Las politicas de igualdad en España: avances y retrocesos. Granada: Universidad de Granada.

GelB, Joyce. 1989. Feminism and Politics: A Comparative Perspective. Berkeley: University of California Press.

GreGORY, Jeanne. 1999. "Gender Mainstreaming: closing the gap between theory and practice», papel presentado en la conferencia Women and Political Action: debating ways forward for feminists. Middlesex University: 18-19 Junio.

Hernes, H. M. 1990. El poder de las mujeres y el Estado de bienestar. Madrid: Vindicación Feminista.

Hoskyns, Catherine. 1999. «Case Study: Mainstreaming gender equality in the European Union", papel presentado en la conferencia Women and Political Action: debating ways forward for feminists. Middlesex University: 18-19 Junio.

Instituto de la Mujer. 1994. 10 Años del Instituto de la Mujer. Madrid: Instituto de la Mujer.
JaHAN, Rounaq. 1995. The Elusive Agenda: Mainstreaming Women in Development. London: Zed Books.

LOVENDUSKI, Joni. 1997. «Feminismo institucional: Género y Estado», en Edurne UriarTe y Arantxa ElizOndo (eds.), Mujeres en Politica. Análisis y práctica. Barcelona: Ariel.

Ministerio de Asuntos Sociales. 1992. Mujer, Protección y Programas Sociales para colectivos de mujeres en riesgo de marginación (1990-1992) Perspectivas. Indicadores de protección social, servicios sociales y programas de igualdad durante el periodo 19821992. Madrid: Ministerio de Asuntos Sociales.

Recipes. 2000. Best Practices of Gender Mainstreaming. http://www.arcidonna.it/mainstreaming/Ligth/inglese/mainstreamingenl.html

Sampedro, María Rosario. 1992. Administracion local y Politicas de Igualdad de la Mujer. Madrid: FEMP (Federación Española de Municipios y Provincias).

SenSAT y VARELla. 1998. «Las políticas dirigidas a las mujeres: la acción pública para la igualdad entre los sexos», en Ricard Gomá y Joan Subirats (eds.), Politicas públicas en España: contenidos, redes de actores y niveles de gobierno. Barcelona: Ariel.

Stetson, Dorothy McBride y Mazur, Amy G. (eds.) 1995. Comparative State Feminism. Thousand Oaks: Sage.

Threlfall, Monica. 1998. «State feminism of party feminism? Feminist politics and the Spanish Institute of Women", The European Journal of Women's Studies 5:6993 .

Valiente FernÁndez, Celia. 1996. «El feminismo institucional en España: el Instituto de la Mujer, 1983-1994", Revista Internacional de Sociología 13: 163-204.

Valiente Fernández, Celia. 1998/1999. "Feminismo de estado en los Ayuntamientos de la Comunidad Autónoma de Madrid», Gestión y Análisis de Politicas Públicas 13/14: 173-189.

Valiente Fernández, Celia. 1999. «El feminismo de Estado y los debates políticos: la formación ocupacional en España", Revista Española de Ciencia Política 1: 127-147. 
